\title{
A systematic review of parent and family functioning in pediatric solid organ transplant populations
}

\author{
Melissa K. Cousino ${ }^{1,2}$ (D) | Kelly E. Rea ${ }^{2}$ ｜Kurt R. Schumacher ${ }^{1,2}$ | John C. Magee ${ }^{2,3}$ | \\ Emily M. Fredericks ${ }^{1,2,4}$
}

${ }^{1}$ Department of Pediatrics, University of Michigan Health System, Ann Arbor, MI, USA

${ }^{2}$ University of Michigan Transplant Center, Ann Arbor, MI, USA

${ }^{3}$ Department of Surgery, University of Michigan Health System, Ann Arbor, MI, USA

${ }^{4}$ Child Health Evaluation and Research Center, University of Michigan, Ann Arbor, MI, USA

\section{Correspondence}

Melissa Cousino, Department of Pediatrics, University of Michigan Health System, Ann Arbor, MI, USA.

Email: melcousi@med.umich.edu

\begin{abstract}
The process of pediatric solid organ transplantation (SOT) places new and increased stressors on patients and family members. Measures of family functioning may predict psychological and health outcomes for pediatric patients and their families, and provide opportunity for targeted intervention. This systematic review investigated parent and family functioning and factors associated with poorer functioning in the pediatric SOT population. Thirty-seven studies were identified and reviewed. Studies featured a range of organ populations (eg, heart, liver, kidney, lung, intestine) at various stages in the transplant process. Findings highlighted that parents of pediatric SOT populations commonly report increased stress and mental health symptoms, including posttraumatic stress disorder. Pediatric SOT is also associated with increased family stress and burden throughout the transplant process. Measures of parent and family functioning were associated with several important health-related factors, such as medication adherence, readiness for discharge, and number of hospitalizations. Overall, findings suggest that family stress and burden persists posttransplant, and parent and family functioning is associated with health-related factors in SOT, highlighting family-level functioning as an important target for future intervention.
\end{abstract}

\section{1 | INTRODUCTION}

Rates of pediatric SOT have increased in prevalence over the last decade with 5-year survival rates exceeding $75 \%$ across pediatric heart and liver transplant populations and $>90 \%$ in pediatric kidney transplant populations. ${ }^{1,2}$ While SOT offers many children and adolescents increased quantity and quality of life ${ }^{3-5}$ patients and families are faced with many stressors and burdens. During the pretransplant phase, patients and families may experience long waits due to the scarcity of donor organs available, ${ }^{2}$ financial challenges, stress on siblings and caregivers as roles and responsibilities shift, and complex medical regimens, all while the child remains seriously ill. ${ }^{6,7}$ Following transplantation, SOT recipients must continue to take daily medications, attend

Abbreviations: PAT, Psychosocial Assessment Tool; PRISMA, Preferred Reporting Items for Systematic Reviews and Meta-analyses; PTSD, posttraumatic stress disorder; SES, socioeconomic status; SOT, solid organ transplantation. frequent follow-up appointments, and undergo various procedures, such as biopsies and cardiac catherizations. As Gold and colleagues ${ }^{8}$ described, parents state that they must "adapt to the new disease called organ transplant," which is accompanied by risks of rejection, graft loss, need for re-transplantation, and mortality. Beyond the stressors of the transplant course itself, children pre- and post-SOT may have complex developmental and emotional needs, which can result in even greater strain on the family system. ${ }^{3,6}$

Thus, it is necessary to consider the impact of SOT on both the family system and the child. Bronfenbrenner's social ecological framework places a child at the center of concentric circles representing various aspects of a child's social ecology, such as parents/family, school, healthcare team/system, community, and socioeconomic class. ${ }^{9}$ Per this framework, parent and family functioning is considered to be critically important with regard to the relationship between a child's development and their disease course. 
A large systematic and meta-analytic review of parents of children with a variety of chronic illnesses supported this notion. Cousino and Hazen ${ }^{10}$ found that parents of children with chronic illnesses experienced greater general parenting stress than parents of healthy children. Although SOT populations were not included in this review, increased parenting stress was found to be associated with poorer child psychological outcomes across disease groups. As a result, parent and family stress has been highlighted as a modifiable intervention target in families of children with chronic illnesses given associations with patient psychological functioning and health-related outcomes. ${ }^{10}$

Similar relationships have been demonstrated in pediatric SOT populations. For example, greater parent and family stress is associated with poorer adherence to post-transplant immunosuppressant medications. ${ }^{11-13}$ This is consistent with studies that have found that parents and adolescents who report healthier family functioning also report fewer medication barriers, such as forgetting medications, scheduling issues, and voluntary resistance of medication administration. ${ }^{14,15}$ As a result, pediatric SOT recipients from healthier functioning family systems experience fewer hospitalizations ${ }^{16}$ and better quality of life. ${ }^{3}$

While investigators have begun to examine parent and family functioning in pediatric SOT populations, far less has been performed when compared to other pediatric illness groups. ${ }^{6}$ The findings to date have not been systematically reviewed and synthesized, which may be attributed to focus on single organ groups and small samples limiting quantitative analysis, among other reasons. Other reviews of this kind have been completed across pediatric chronic illness groups, including oncology, ${ }^{17}$ diabetes, ${ }^{18}$ and chronic pain, ${ }^{19}$ among others. While similarities are expected among pediatric SOT populations and these other illness groups given the chronicity of SOT, differences in life expectancy, treatment regimen demands, and unknown timing of organ availability, among others, are likely to impact SOT families in unique ways.

To address this gap in the literature and guide the development of evidence-based interventions, this study aimed to review and summarize the literature regarding family functioning among pediatric SOT patients and their families. Guided in part by the social ecological framework, ${ }^{9}$ the current study aimed to answer the following questions: (i) What is the impact of pediatric SOT on parent psychological functioning? (ii) What is the impact of pediatric SOT on family functioning? and (iii) What variables are associated with poorer parent and family functioning in the pediatric SOT population? It is our objective that answers to these questions will help to identify modifiable familybased intervention targets in pediatric SOT populations.

\section{2 | METHODS}

\subsection{Search strategy}

Literature searches were conducted on the following databases: PsychInfo, PubMed, MEDLINE, and Cumulative Index to Nursing and Allied Health Literature, and the Cochrane Systematic Review and Controlled Trials Database. In an effort to provide an extensive review of the literature while also limiting the review to studies most relevant to current medical practice, the search included articles published in peer-reviewed journals from 1980 to 2016. Databases were searched using the following word stems: (i) "child \$\$," "youth," "adolescen $\$$," "teen\$," "infant," "pediatric," "paediatric," (ii) "organ," "transplant," "solid organ transplant," (iii) "parent," "mother," "father," "caregiver," "family," "system," and (iv) "depression," "anxiety," "trauma," "stress," "distress," "marital," "functioning," "coping," and "adaptation." The reference sections of articles meeting the predefined inclusion criteria were examined for additional studies reporting on parent and family functioning in pediatric SOT populations. Manual searches of the Journal of Pediatric Psychology and Pediatric Transplantation were also conducted.

\subsection{Inclusion criteria}

In accordance with Cochrane Collaboration guidelines, ${ }^{20}$ the following inclusion criteria were defined prior to initiating the literature search: (i) publication date between 1980 and 2016; (ii) publication in a peerreviewed journal; (iii) published in the English language; (iv) included a study sample of pediatric (0-21 years) SOT populations, including heart, lung, kidney, liver, intestinal, and multivisceral transplant populations, either pre- or post-organ transplantation; and (v) included an objective measure of parent report of psychological, family, or marital functioning. Initially, the authors aimed to complete a meta-analytic review; however, search results yielded an insufficient number of studies with comparison group data and/or data needed for the computation of raw effect statistics for between-groups comparisons. Studies specific to sibling functioning only were not included in this review.

\section{3 | Data extraction and study coding}

Each included study was coded for patient and family outcomes and evaluated for potential bias by the first two authors ( $M C$ and $K R$ ). Data extracted from each study included transplant sample characteristics (organ population, pre-/post-transplant, age), parent and family characteristics, use of a comparison group, assessment measures, and overall findings. Sample size, control group comparisons, use of established measurements, multimodal and multi-informant assessment, and data attrition, including missing, lost, or excluded data, were all considered when assessing studies for risk of bias. Bias analysis revealed that in all of the studies, a minimum of at least one parentcompleted questionnaires was used. Although few authors included psychometric data in their manuscripts, all of the studies included use of at least one commonly used, valid and reliable measure of either psychological, family, or marital functioning.

\section{3 | RESULTS}

\section{1 | Study characteristics}

Following the PRISMA guidelines, our search yielded 617 studies, after excluding duplicate studies $(n=61)$. See Figure 1 for the PRISMA flow diagram. Of the studies excluded, the majority did not include a pediatric SOT population or did not utilize a quantitative measure of 
parent and/or family functioning. The 37 studies meeting inclusion criteria were further reviewed, and data were extracted according to our predetermined questions of interest. Within these 37 studies, year of publication ranged from 1988 to 2015 , with approximately half of the studies published more than 10 years ago $(n=18)$, and 11 studies published within the last 5 years. Many studies $(n=12)$ included SOT patients across multiple organ groups. Eleven of the included studies examined only kidney transplant populations, followed by nine studies looking at only liver transplant populations. Five studies included heart transplant patients only. The overwhelming majority of included studies examined only pediatric patients post-transplant $(n=27)$, as compared to only pretransplant patients $(n=6)$ or both pre- and posttransplant patients $(n=4)$. Included studies examined pediatric patients within the United States ( $n=27)$, as well as other countries: Japan $(n=3)$, Germany $(n=1)$, Canada $(n=1)$, United Kingdom $(n=1)$, Norway $(n=1)$, Switzerland $(n=1)$, Argentina $(n=1)$, and Australia $(n=1)$.

\subsection{What is the impact of pediatric SOT on parent psychological functioning?}

\subsection{1 | Parent psychological functioning}

Results are summarized in Table 1. Findings specific to parent psychological functioning were found to be inconclusive across the literature.
For example, in a study of 86 mothers and 58 fathers of children prekidney transplant, scores on a commonly used adult depression measure were predominately in the minimal to mild range, with $<6 \%$ of mothers endorsing severe symptoms. ${ }^{21}$ Consistent with this finding, in a sample of mothers of 14 children ages 3-8 who underwent a kidney transplant in the past 3 years, mean scores on a parent-completed global mental health rating scale were in the average range. ${ }^{22}$ Others have reported similar findings when assessing depression and anxiety in parents post-heart, kidney, and liver transplant. ${ }^{23}$

However, contrary to these findings, in a sample of 61 parents (41 mothers and 20 fathers) of children pre- or 2 months post-liver and/or intestinal transplantation, $51 \%$ of parents reported clinically significant psychological symptoms on a global distress rating scale. There were no differences observed with regard to time at assessment (ie, pre- or post-transplantation). ${ }^{24}$ Similar findings were reported by Diseth and colleagues ${ }^{25}$ in a post-kidney transplant population, noting that mothers' reports of mental health problems were greater than healthy controls and similar to mothers of children with leukemia. Simons and colleagues found that mothers of pre-SOT patients reported greater global distress than normative populations with those mothers of listed patients reporting greater distress than those who were not listed. ${ }^{26}$ Although Douglas and colleagues reported mean scores within the average range for mothers of kidney transplant recipients, over $50 \%$ of fathers reported clinically significant mental
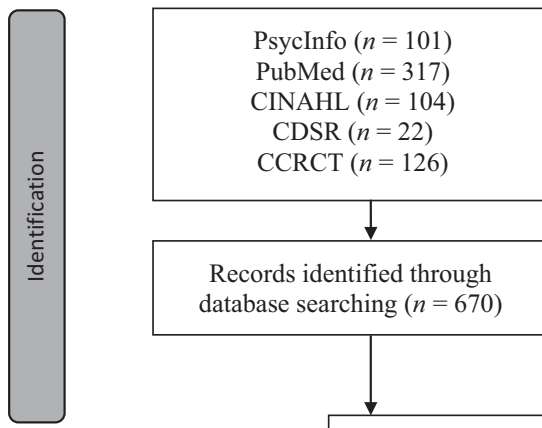

Records identified through database searching $(n=670)$
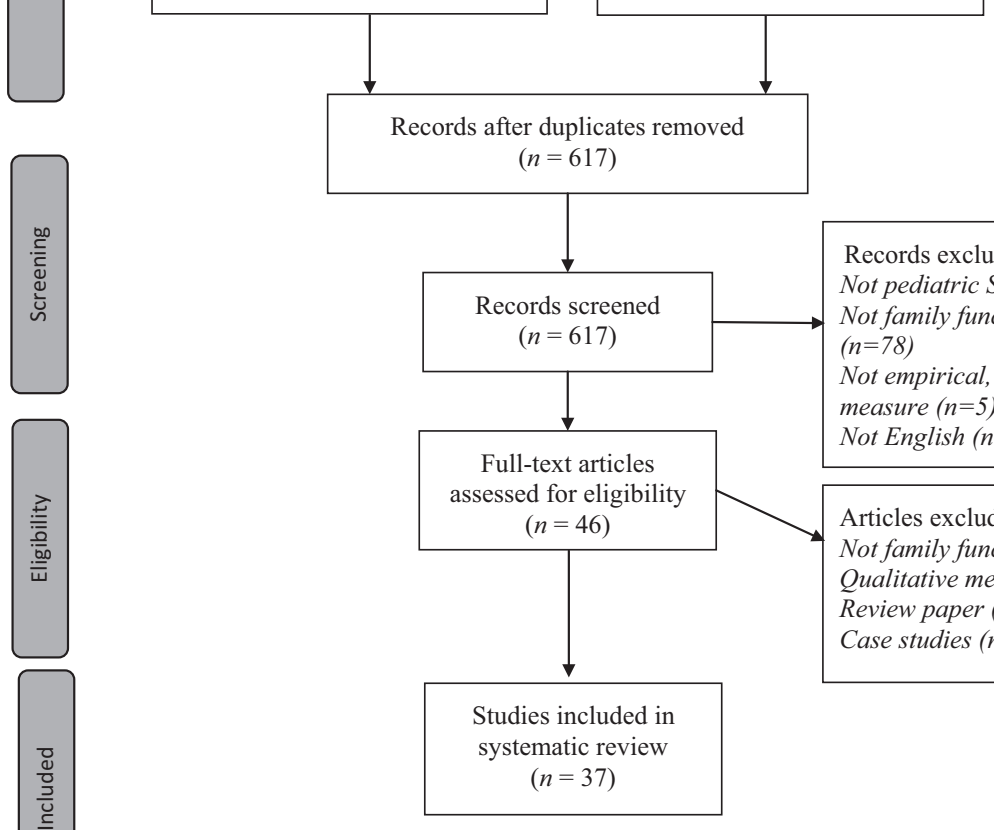

Studies included in systematic review $(n=37)$

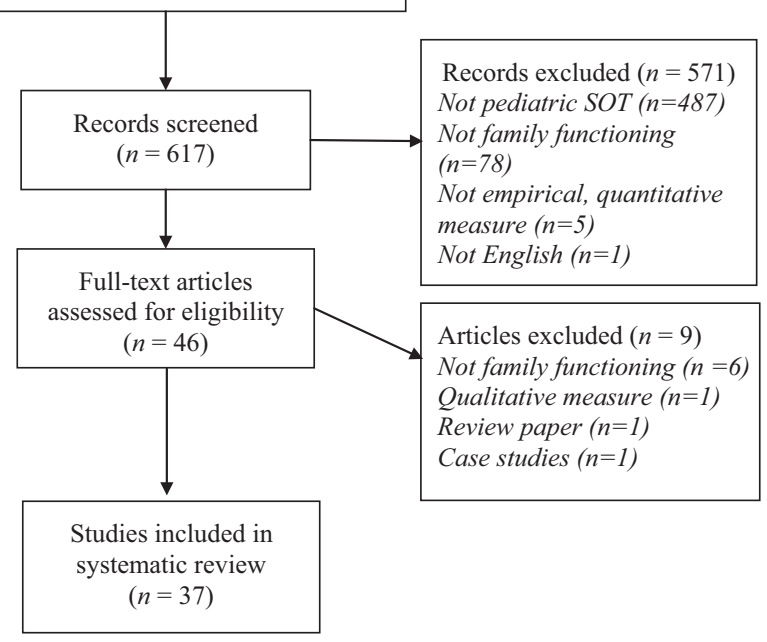




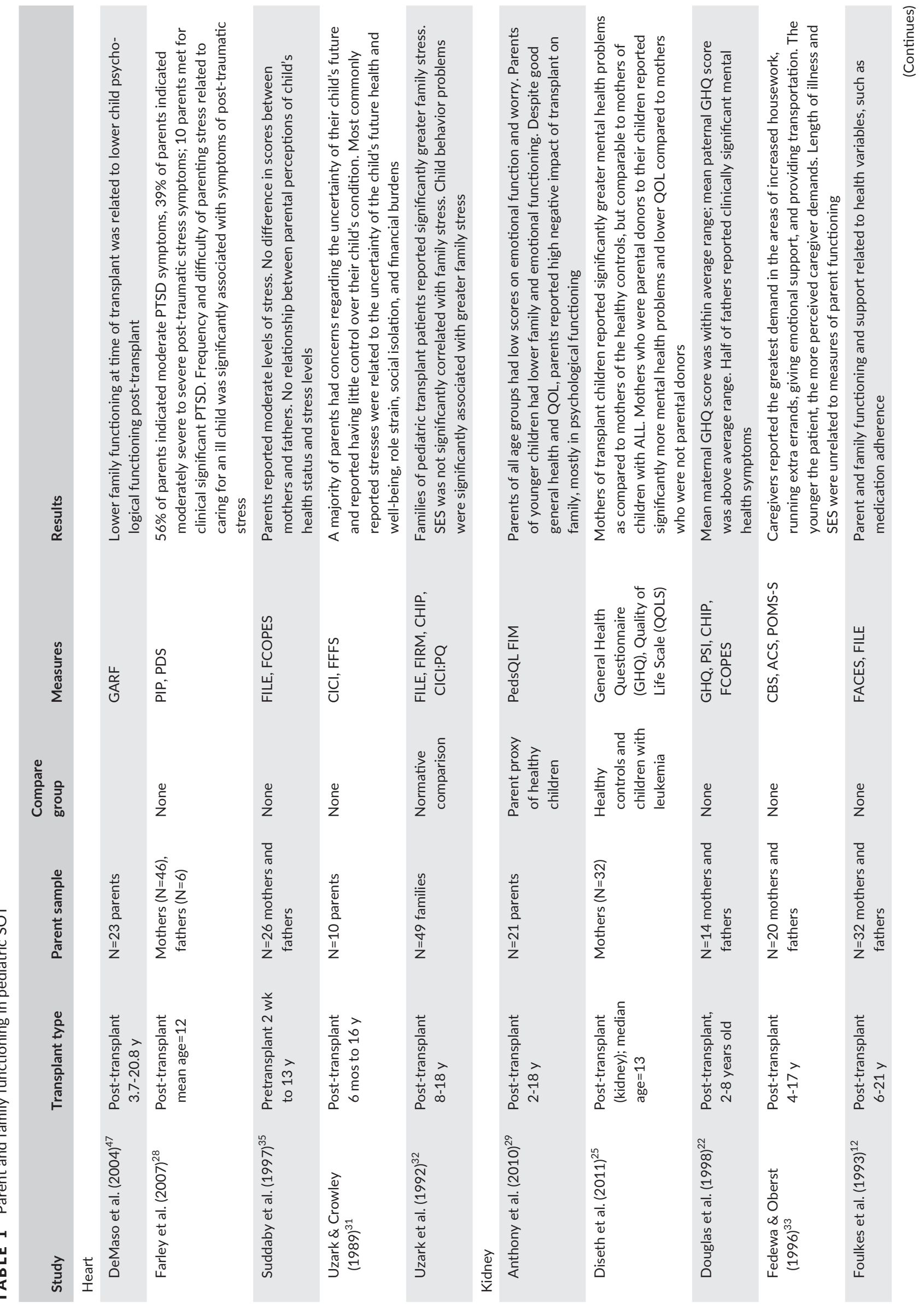



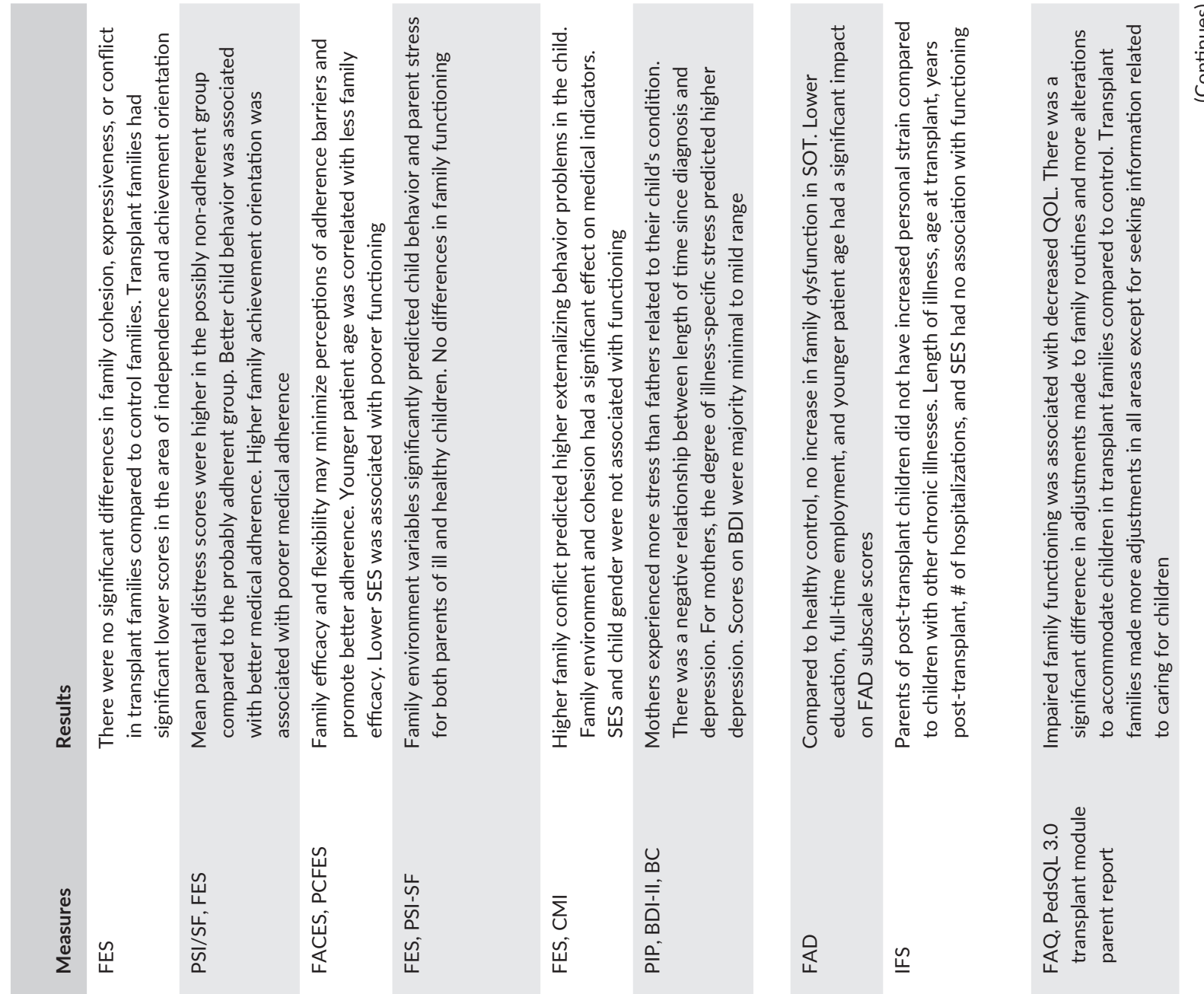

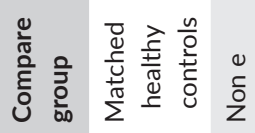

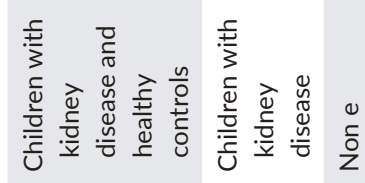

品嵒

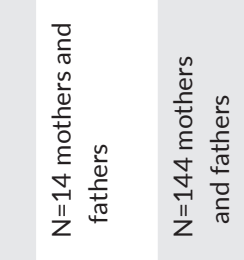

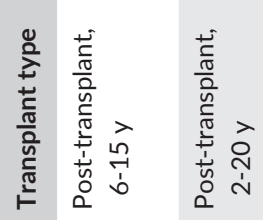
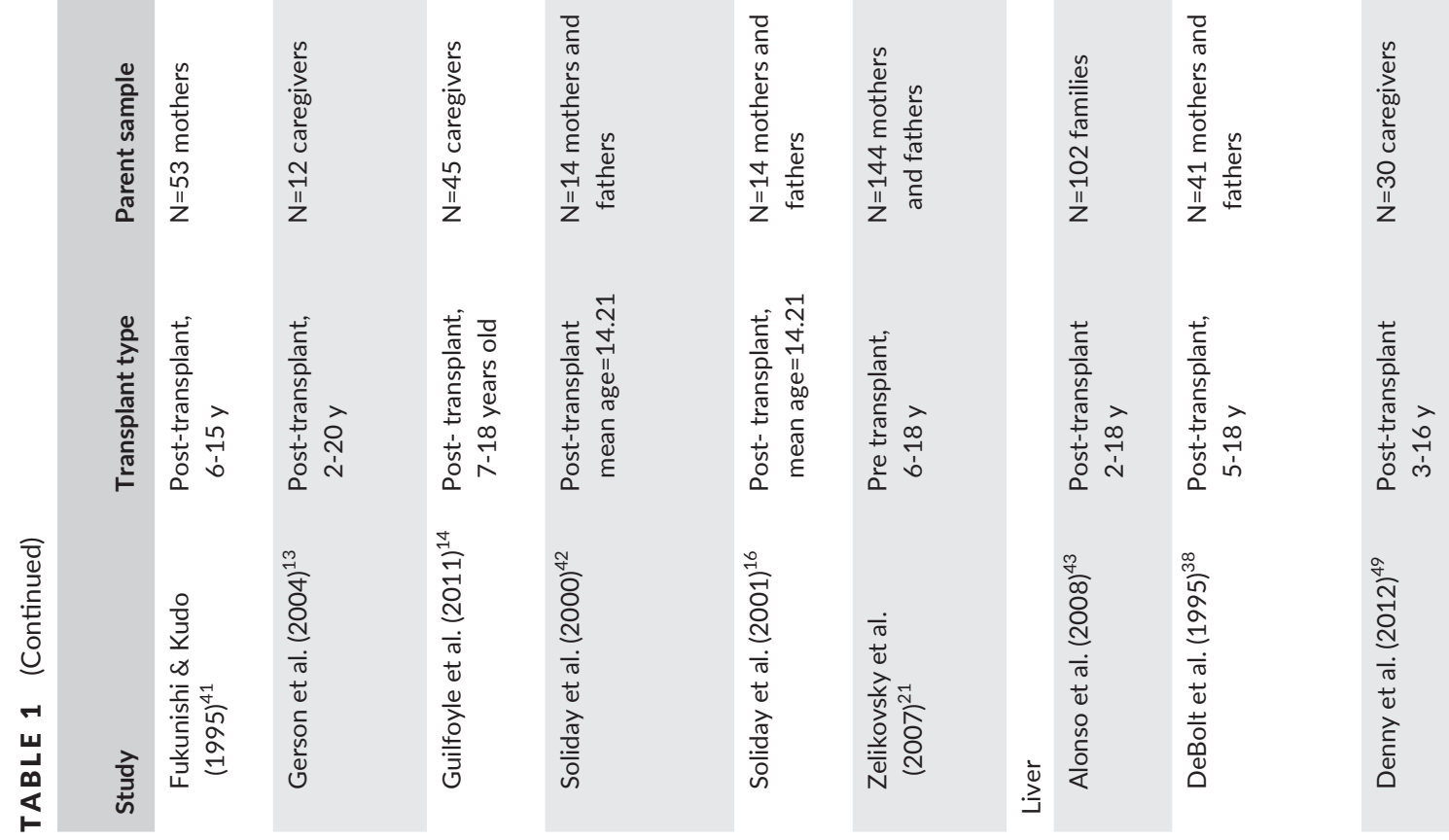


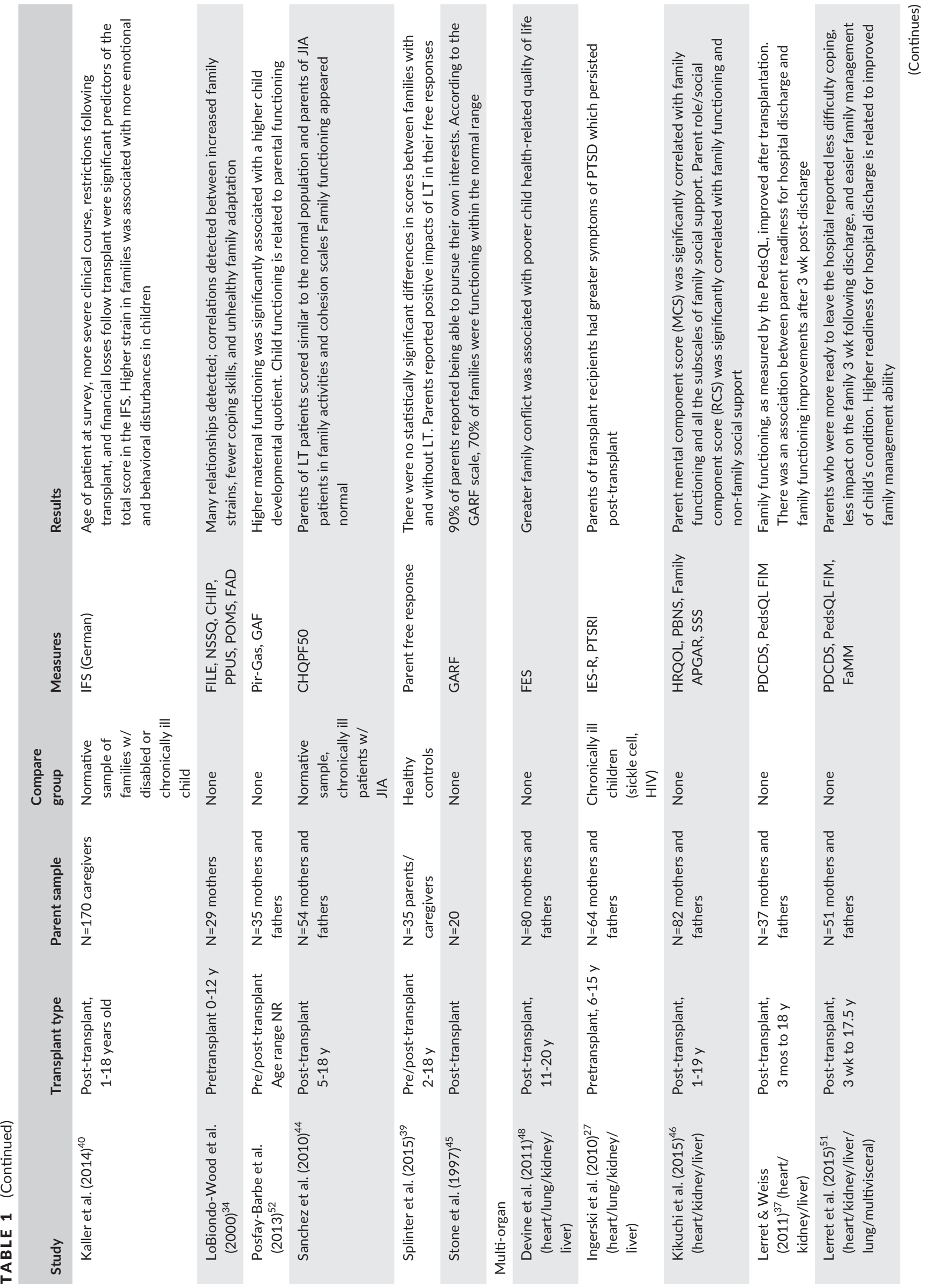



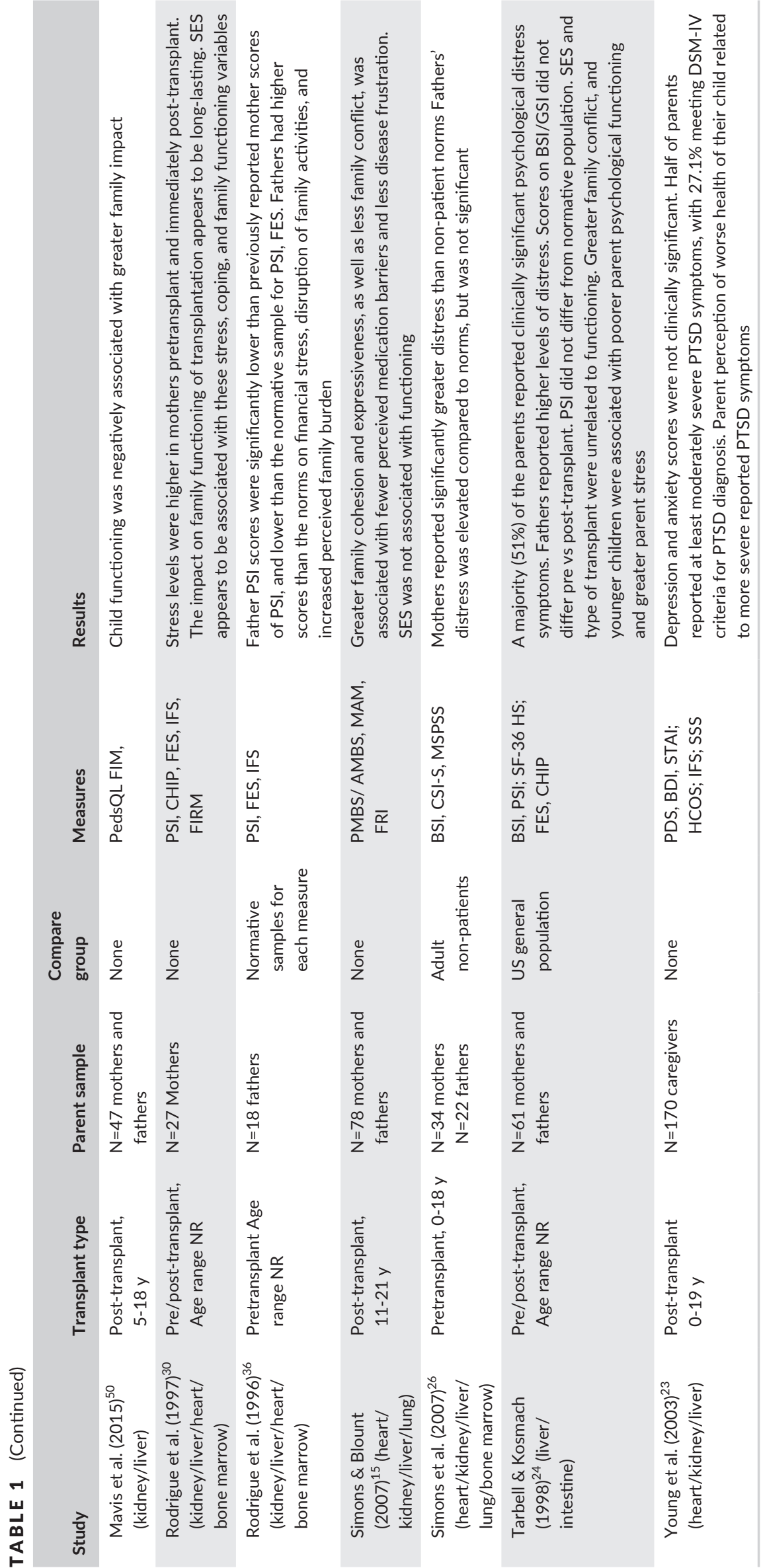

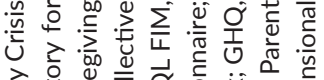

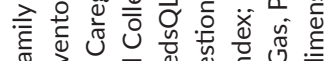

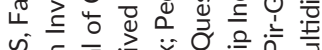

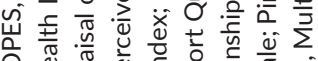
论零 is.

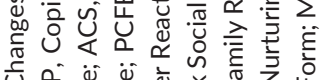

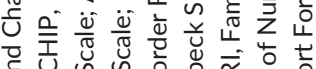

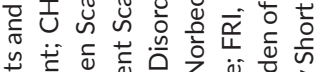

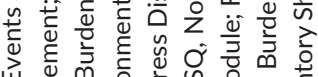

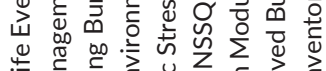

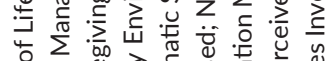

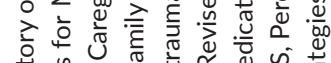

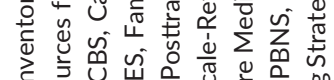

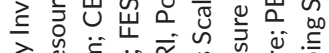

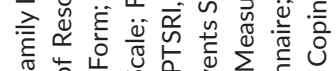

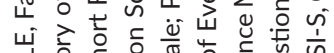

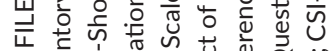

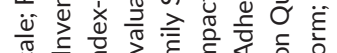
出

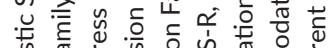

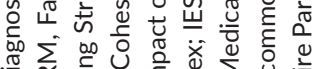

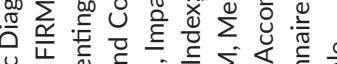

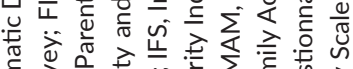

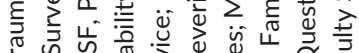

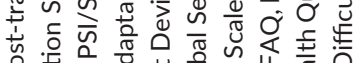

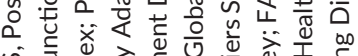

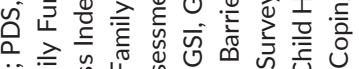

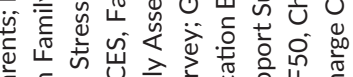

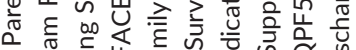
흥ㄷㄹ

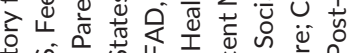

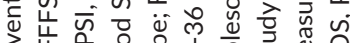

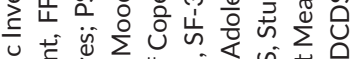

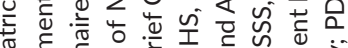

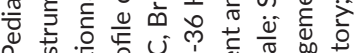

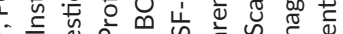

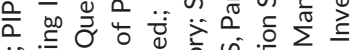

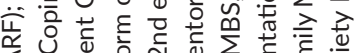

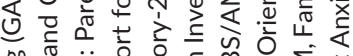

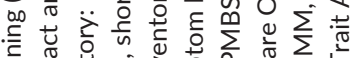

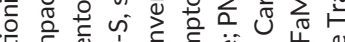

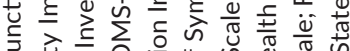

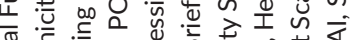
๙

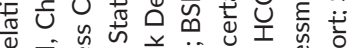

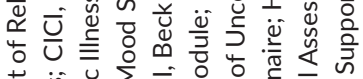

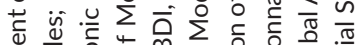

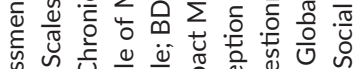

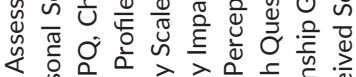

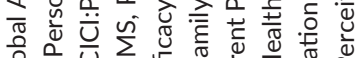

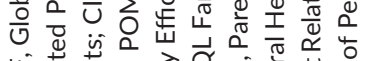

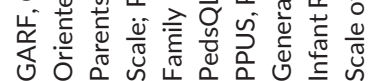


health symptoms. ${ }^{22}$ Others have also reported that fathers endorsed greater psychiatric distress, such as depression and obsessive compulsive symptoms, when compared to mothers. ${ }^{24}$ However, this finding has not been conclusively replicated across the literature. ${ }^{21}$

Rates of PTSD have also been found to be high in parents of SOT candidates and recipients. When compared to other chronic illness populations (ie, HIV and sickle cell), parents of children undergoing evaluation for transplantation (ie, solid organ and bone marrow) reported greater symptoms of PTSD. ${ }^{27}$ Symptoms of parental PTSD may also persist years post-transplantation. In a study of 170 parents, $50.6 \%$ of the sample reported moderate levels of post-traumatic stress symptoms. Per DSM-IV criteria, nearly one-third of the sample met criteria for PTSD. ${ }^{23}$ Similar findings were reported by Farley and colleagues. $^{28}$

\subsection{2 | Parenting stress}

Although Tarbell and Komasch ${ }^{24}$ found that general parenting stress in the months following SOT was similar to healthy comparison groups, others have reported that moderate to high levels of parenting stress and burden continue beyond the pretransplant phase and post-surgical hospitalization. ${ }^{29-32}$ In a cross-transplant population (including liver, kidney, heart, and bone marrow recipients), parenting stress was greatest at 1 month post-transplant with $56 \%$ of mothers reporting clinically significant levels of parenting stress. Forty-one percent of mothers continued to report similar levels of parenting stress 6 months post-transplant. ${ }^{30}$

This may be a result of sustained stressors and worries. For example, in a sample of 10 parents of children 3-24 months post-heart transplantation, $89 \%$ of participants endorsed high amounts of stress related to the uncertainty of their child's future and extra demands on time/energy. ${ }^{31}$ Similarly, in a sample of 20 parents of children ages 4-17 years who were post-kidney transplant, respondents stated that increasing housework, providing emotional support, and managing behavior problems were their most difficult tasks, while monitoring for signs of a rejection was a time-consuming task. ${ }^{33}$ Many parents (89\%) also described feeling as although they had little control over their child's condition. ${ }^{31}$ Nearly a third of mothers of young kidney transplant recipients perceived that others blamed them for the child's health issues, while $57 \%$ blamed themselves. ${ }^{22}$

\subsection{What is the impact of pediatric SOT on family functioning?}

\subsection{1 | Family stress and burden}

Researchers have also examined parental report of overall family stress and burden. In the pretransplant evaluation phase, mothers of liver transplant candidates reported high family stress. ${ }^{34}$ Similarly, $77 \%$ of parents $(\mathrm{N}=26)$ of children actively listed for heart transplantation endorsed family stress levels greater than population norms. ${ }^{35}$ Consistent with these findings, in a sample of only fathers of children being evaluated for transplantation (ie, liver, kidney, heart, or bone marrow), respondents described greater financial stress, family burden, and disrupted planning as a result their child's illness when compared to the normative sample. ${ }^{36}$

Findings from Lerret and Weiss ${ }^{37}$ suggest that families may experience a decrease in burden from the day of hospital discharge to 3 weeks post-discharge. In a sample of 41 parents whose child underwent liver transplantation $\geq 4$ years ago, negative impact of illness on the family system was reported to be less than other pediatric chronic illness groups. ${ }^{38}$ Findings, however, are not consistent across the literature. For example, in a small cross-transplant longitudinal study, family burden, financial burden, and caretaker burden were greater in the post-transplant period when compared to assessments conducted during pretransplant evaluations. ${ }^{30}$ Splinter and colleagues recently demonstrated that family impact of disease is similar in families of children post-liver transplant and those of children living at home with other chronic conditions. ${ }^{39}$ Kaller and colleagues also found that parents of liver transplant recipients, with a mean time since transplant of 5.8 years, reported that the burdens associated with their child's condition caused greater financial impact, impact on family coping, and impact on siblings when compared to a sample of families of children with other chronic illnesses/disabilities. ${ }^{40}$ These results have been replicated in parents of kidney transplant recipients who endorsed high levels of family burden post-transplant, particularly in the areas of emotional functioning and worries. ${ }^{29}$

\subsection{2 | Family functioning}

Fewer studies have described the relationships between pediatric SOT and family functioning. In a mixed SOT group, family conflict was greater at 6 months post-transplant when compared to 1 month post-transplant. ${ }^{30}$ Overall, however, the limited work in this area suggests that family functioning in pediatric SOT populations is similar to healthy controls. For example, in a Japanese sample of children both pre- and post-kidney transplant, there were no differences reported in family cohesion, expressiveness, and conflict when compared to healthy controls. ${ }^{41}$ Similar findings were reported in a small US sample of kidney transplant recipients ${ }^{42}$ and in three studies involving liver transplant recipients. ${ }^{43-45}$

\subsection{What variables are associated with poorer parent and family functioning in the pediatric SOT population?}

\subsection{1 | Family factors}

Mixed findings have been reported with regard to family SES with some studies demonstrating associations between lower SES and poorer parent and family functioning, ${ }^{14,17,21}$ and others citing no associations. $^{24,26,32,33,38,42}$ Parental education and marital status were unrelated to parenting stress and depressive symptoms in a large sample of mothers and fathers of children prekidney transplant. ${ }^{21}$

Greater family conflict ${ }^{24}$ and illness-specific parenting stress ${ }^{21}$ have been found to be associated with poorer parental psychological 
functioning. A similar relationship between unhealthy family function and decreased parental emotional and physical quality of life was detected in a Japanese post-SOT population. ${ }^{46}$ Parents who endorse lower family functioning at time of transplant are more likely to report deficits in family functioning years post-transplant. ${ }^{47}$

\subsection{2 | Child factors}

Associations between younger child age at time of assessment with greater parenting stress, ${ }^{24,29,40}$ caregiver demands, ${ }^{33}$ and less family efficacy for completing necessary tasks ${ }^{14}$ have been reported. However, two studies were unable to detect relationships between child age, parent psychological functioning, parenting stress, and family functioning. ${ }^{26,30}$ In one study, parents of female kidney transplant recipients reported better family communication and efficacy when compared to their male counterparts. ${ }^{14}$ Others have found no relationship between child gender and parent-reported stress or depressive symptoms in pretransplant populations. ${ }^{21,26}$

With regard to child psychological functioning, greater family conflict was associated with increased externalizing behavioral problems in children post-kidney transplant ${ }^{16}$ and poorer child health-related quality of life in a mixed SOT population. ${ }^{48}$ Similarly, greater family stress positively correlated with increased child emotional and behavioral problems in post-liver ${ }^{40}$ and post-heart transplant populations. ${ }^{32}$ Fewer adjustments to family routines and lifestyle (eg, moving homes, increasing supervision of child during play) following liver transplantation were associated with better child quality of life. ${ }^{49}$

\subsection{3 | Health-related factors}

In addition to family and child factors, health-related correlates of parent and family functioning have also been investigated. Type of transplant was unrelated to parent psychological functioning ${ }^{24}$ and parent-reported levels of PTSD. ${ }^{23}$ Time since diagnosis was unrelated to parenting stress and depressive symptoms in a prekidney transplant population. ${ }^{21}$ Similarly, in samples of post-kidney (2-14 months) and liver ( $\geq 4$ years) transplant recipients, child length of pretransplant illness, age at transplant, years post-transplant, and number of hospitalizations were unrelated to caregiver and family burden. ${ }^{33,38}$ In accord with the above findings, length of transplant hospitalization was unrelated to parenting stress and family functioning in a crosstransplant population. ${ }^{30}$

Parental perception of their child's illness severity was unrelated to parenting stress in a heart transplant population. ${ }^{35}$ Similarly, child adaptive functioning/functional status was unrelated to parental psychological functioning and general parenting stress in the pre- and perioperative transplant phases in another study. ${ }^{24}$ Others have reported discordant findings with regards to family impact of disease and child functional status ${ }^{50}$ and clinical course severity. ${ }^{40}$ Consistent with the broader pediatric literature, poorer child physical health was associated with increased parent PTSD symptoms. ${ }^{23}$ Likewise, if parents perceived their child to be more vulnerable post-SOT, family impact of disease was greater. ${ }^{50}$
Notably, across multiple studies, parent and family functioning was found to be related to important health-related variables, such as adherence to immunosuppressant medications. ${ }^{12,13}$ For example, in 13 post-kidney transplant recipients, greater general parenting stress was associated with poorer adherence to immunosuppressant medications per physician review of serial laboratory levels. ${ }^{13}$ Consistent with these findings, greater familial efficacy and flexibility have been shown to be related to fewer perceived medication adherence barriers. ${ }^{14}$ Greater family cohesion and expressiveness, as well as less family conflict, are also associated with fewer adolescent reported medication barriers and lower disease frustration. ${ }^{15}$

In addition to medication-related outcomes, parent and family functioning has been found to be associated with readiness for hospital discharge ${ }^{51}$ and number of hospitalizations. In a mixed sample of children with kidney disease, including those with end-stage renal disease and post-transplant recipients, less family cohesiveness was associated with greater number of hospitalizations, accounting for $10.24 \%$ of the variance. ${ }^{16}$ Although no studies reviewed investigated relationships between parent and family functioning and graft survival, healthier maternal psychological functioning was positively correlated with better psychomotor development in a liver transplant population, comprising 21 children from seven different countries. $^{52}$

\section{4 | DISCUSSION}

To our knowledge, this was the first study to systematically review and synthesize the research on parent and family functioning in pediatric SOT populations. Reviews of this nature are important, providing an accessible integration of the literature to assist in guiding future research efforts, while also identifying inconsistencies and gaps in the science to date. Consistent with findings across the pediatric chronic illness literature ${ }^{10}$ results of this systematic review suggest that parents of children pre- and post-SOT endorse significant parenting stress and burden. Our findings are also consistent with those reported across the adult SOT literature where high rates of caregiver psychiatric illness ${ }^{53}$ and caregiver strain ${ }^{54}$ have been documented well beyond the pre- and immediate post-transplant periods.

Furthermore, although findings were inconsistent across some studies, results of this review suggest that parents of pediatric SOT patients are at increased risk for depression and PTSD. For example, Young and colleagues found that one-third of parents of children postSOT met criteria for a diagnosis of PTSD ${ }^{23}$ compared to only $3.5 \%$ of adult community samples meeting criteria for current PTSD. ${ }^{55}$ Rates of parental PTSD among pediatric SOT populations are similar to those of pediatric oncologic populations. ${ }^{56}$ This review also identified consistent findings demonstrating an association between parent and family functioning and child health-related factors, such as adherence, fewer medication barriers, and number of hospitalizations. Although the direction of this association is unknown per the current literature, findings are concordant with those across other childhood chronic illness populations. $^{57-59}$ 
Given associations between parent and family functioning and child health-related factors, it is critically important that we seek to identify correlates of poorer parent and family functioning, as these may serve as modifiable intervention targets. Interestingly, no family demographic factors were conclusively identified as correlates of parent and family functioning. For example, only three studies detected an association between family SES and parent and family functioning, ${ }^{14,21,30}$ while a number of studies reported a null relationship between the variables. ${ }^{15,16,33}$ To date, this literature has not thoroughly investigated other parent and family factors that have been identified to increase risk of poorer parental psychological outcomes in other pediatric illness groups. For example, as suggested by Mavis and colleagues ${ }^{50}$ and findings across other pediatric illness groups, ${ }^{10}$ it may be that parental cognitive appraisals (eg, perceived vulnerability of child, parental self-efficacy regarding disease management), best explains why some parents of pediatric SOT patients are at greater risk for poorer psychological outcomes.

Similarly, no transplant-specific factors (eg, type of transplant, time since transplant) were associated with parent and family functioning. Others have reported null relationships between illness duration and parent and family psychosocial outcomes among other pediatric illness populations. ${ }^{10}$ Again, it may be that important health-related variables have been overlooked by the transplant literature to date. For example, review of the larger pediatric chronic illness literature suggests that parents with greater responsibility for the child's treatment regimen report greater stress and burden. ${ }^{10}$ Thus, although differences in etiology, treatment course, and survival rates are present across the organ groups, findings underscore the importance of screening all families, regardless of organ type or other transplant-related factors, until health-related risk factors are better understood.

Across the literature, younger child age at time of assessment was associated with more negative parent and family sequelae. This may in part be due to the fact that parents of younger children take primary responsibility for the complex medical management of SOT patients. Younger children are also more likely to experience greater procedural distress and medically associated fears; ${ }^{60,61}$ therefore, the frequent blood draws and appointments may be difficult for parents as they regularly see their child in distress. In addition, parents of younger children may be newer to the demands of parenting or with their first child. Researchers have reported similar findings in parents and families of children with diabetes, ${ }^{62}$ cancer, ${ }^{63}$ and other chronic illnesses. ${ }^{64}$ Child emotional and behavioral problems were also associated with poorer parent and family functioning. ${ }^{16,40}$ Although the direction of this relationship is unclear, parents reporting child psychological problems may also benefit the most from parent- or family-directed interventions as well.

\subsection{Limitations of the literature and future directions for research}

Overall, this literature is limited by small sample sizes; thus, results must be interpreted with caution. Many studies had fewer than 30 participants. Most studies were conducted at single centers and combined various transplant groups (ie, pre- and post-transplant, organ types, SOT, and stem cell). In addition, studies span multiple decades and significant advancements have been made in SOT and survival rates throughout this vast time span. These may explain the many inconsistent findings across the literature. Some studies may have been underpowered to detect associations, while others may have included too diverse of participants. For example, heart, lung, and liver transplant patients do not have long-term alternative treatments available, whereas kidney transplant patients can be maintained on dialysis for years until a suitable organ becomes available and/or in the instance of disease re-occurrence.

Secondly, the majority of research in this area has been carried out in pediatric kidney and liver transplant populations. Very few studies investigating parent and family functioning in heart, lung, intestinal, and multivisceral populations were identified. In addition, studies used a variety of assessment measures to assess a number of different domains relevant to parent and family functioning. These differences in measurement selection and constructs of interest likely contributed to the inconsistent and discordant findings among studies. Selection bias is also of potential concern as parents who were more or less stressed may have been more agreeable to participating in the studies. Lastly, much of the work to date has been cross-sectional in nature limiting our ability to determine causality. Based upon the current literature, we cannot conclude that pediatric SOT causes increased parent and family distress. Nevertheless, it is apparent that families of children with SOT report higher levels of family stress and burden, which is worthy of further investigation.

Thus, with regard to future directions for research, longitudinal investigations are needed to better understand relationships and causality, identify times of greatest risk for parent distress and family dysfunction, and determine the long-term impact of parent and family functioning on patient health-related outcomes. In addition, familyand disease-specific factors that may impact parent and family distress should be further explored. Distance from hospital and family size are two important family-related variables that have not yet been explored. Furthermore, are parents of children with genetically inherited diseases, such as familial dilated cardiomyopathy, at greater risk due to feelings of guilt or perceptions that they "caused" transplantation for their child? Diseases with high rates of re-occurence, such as focal segmental glomerulosclerosis, may also cause greater stress and burden on family systems, as could the prospect of re-transplantation, which is imminent in some organ groups.

In addition, while associations between parent and family functioning and some patient psychosocial and health-related outcomes have been examined, additional work in this area is needed. Only two studies have tested relationships between parent and family functioning and patient adherence to treatment regimen. Research in other chronic illness groups suggests that parent and family functioning strongly predicts adherence outcomes. ${ }^{57,58}$ Other health-related outcomes as they relate to parent and family functioning, such as graft survival, readiness for transition to adult care, involvement in medical decision-making, and health-related quality of life should also be explored. 


\section{2 | Clinical implications}

Clinically, results of this review underscore the value of assessing parent and family functioning as part of regular pre- and post-transplant care given associations with patient health-related outcomes. It is important to note that not all stress is abnormal and actionable. Pediatric transplantation is indeed an understandably stressful intervention, and some degree of worry, burden, and impact on the family system is expected. However, it remains critically important to identify those parents and families with clinically significant psychosocial impairments.

A number of brief parent and family screening measures exist to assist providers in identifying these parents and families. Measures used across this literature vary greatly. Researchers used measures of either parental psychological functioning (symptoms of depression, PTSD, etc.) or family functioning. Measures of family functioning included assessment of general and illness-specific family stress, overall family functioning, and changes in family routines, among others. None of the measures used in the articles reviewed assess both parent psychological and family functioning in one tool. From a research standpoint, use of construct specific measures (eg, parental depression vs family stress) can yield greater clarity; however, in clinical practice, it is often most helpful to utilize brief screening measures that can be quickly administered and reviewed. The PAT, which is comprised of seven subscales (ie, Family Structure and Resources, Social Support, Child Problems, Sibling Problems, Family Problems, Parent Stress Reactions, and Family Beliefs), has been validated for use in pediatric SOT populations. ${ }^{65,66}$ Use of a brief screening tool, such as the PAT, helps to identify parents and families in greatest need of additional intervention. Upon reviewing the PAT, more specific measures based upon areas of identified risk, like those used in the reviewed articles, can then be utilized.

Upon identifying those at greatest risk, it is necessary that appropriate follow-up intervention then be provided. For parents endorsing symptoms of depression or PTSD, for example, referral for local therapy and/or psychopharmacological evaluation may be necessary. For those reporting high rates of family stress or disruptions to family routines, intervention may include in-clinic problem-solving and psychoeducation provided by transplant-affiliated mental health professionals, such as psychologists and social workers. Given the limitations of what can be provided during transplant clinic settings, group-based interventions that serve a larger number of families in need may be particularly fruitful. Kazak and colleagues developed a 1-day familybased group cognitive behavioral intervention for those affected by childhood cancer. ${ }^{67}$ This brief intervention aimed to decrease parent and family distress and improve family functioning. Participants reported decreases in parental anxiety and PTSD, which were sustained 6 months following participation in the group. This program could be adapted to meet the unmet needs of parents and families of the pediatric SOT population; however, concurrent investigation of its effectiveness through the conduct of randomized controlled trials would be also needed to best determine the intervention's impact on family and child psychosocial outcomes, as well as child health-related outcomes.

\subsection{Study limitations}

Results of this review should be considered in light of our own study limitations. Although efforts were made to identify all relevant research, some studies may not have been identified and included in this review. Search terms were broad in an attempt to capture the many ways one may refer to parent and family functioning; however, given great variation in terminology used, studies meeting inclusion criteria may not have come up in the database searches. Furthermore, in the reviewed articles, authors use an array of terms to describe and measure family functioning (eg, family stress, family burden, family distress). Without clear definitions and/or concurrent validity tests among all of these measures, it is unclear how similar or dissimilar each construct of interest is. The inclusion criteria were also limited to studies including a quantitative measure of parent and/or family functioning. Therefore, notable qualitative studies that have highlighted issues important to understanding parent and family functioning in pediatric SOT, such as work by Mendes and Bousso, ${ }^{68}$ Chou and colleagues, ${ }^{69}$ and Williams and colleagues, ${ }^{70}$ were not included in this review.

Despite these limitations, this first systematic review of its kind provides a helpful synthetization of the pediatric SOT literature and highlights necessary next steps for action. Given the high rates of parental and family psychological distress, and their impact on child health and psychosocial outcomes, it is imperative that greater attention be given to screening and intervening upon parent and family stressors during both the pre- and post-transplant period. Further research is needed to determine whether or not interventional efforts of this nature have the potential to improve long-term graft and patient survival of pediatric SOT populations.

\section{AUTHORS' CONTRIBUTIONS}

Melissa K. Cousino: Concept/design, data analysis/interpretation, drafting article, critical revision of article, and approval of article; Kelly Rea: Data analysis/interpretation, drafting article, critical revision of article, and approval of article; Kurt R. Schumacher: Concept/design, drafting article, critical revision of article, and approval of article; John C. Magee: Concept/design, critical revision of article, and approval of article; Emily M. Fredericks: Concept/design, data analysis/interpretation, critical revision of article, and approval of article.

\section{REFERENCES}

1. Workman JK, Myrick CW, Meyers RL, Bratton SL, Nakagawa TA. Pediatric organ donation and transplantation. Pediatrics. 2013;131:1723-1730.

2. Organ Procurement and Transplantation Network. Survival by patient age. 2016. http://optn.transplant.hrsa.gov

3. Fredericks EM, Zelikovsky N, Aujoulat I, Hames A, Wray J. Post-transplant adjustment-the later years. Pediatr Transplant. 2014;18:675-688.

4. Taylor R, Franck LS, Gibson F, Dhawan A. A critical review of the health-related quality of life of children and adolescents after liver transplantation. Liver Transplant. 2005;11:51-60. 
5. Uzark K, Griffin L, Rodriguez R, et al. Quality of life in pediatric heart transplant recipients: a comparison with children with and without heart disease. J Heart Lung Transpl. 2012;31:571-578.

6. Shellmer D, Brosig C, Wray J. The start of the transplant journey: referral for pediatric solid organ transplantation. Pediatr Transplant. 2014;18:125-133.

7. Aldridge MD. How do families adjust to having a child with chronic kidney failure? A systematic review Nephr Nurs J. 2008;35:157-162.

8. Gold LM, Kirkpatrick BS, Fricker FJ, Zitelli BJ. Psychosocial issues in pediatric organ transplantation: the parents' perspective. Pediatrics. 1986;77:738-744.

9. Bronfenbrenner U. Contexts of child rearing: problems and prospects. Am Psychol. 1979;34:844-850.

10. Cousino MK, Hazen RA. Parenting stress among caregivers of children with chronic illness: a systematic review. J Pediatr Psychol. 2013;38:809-828.

11. Fredericks EM. Family roles and routines after pediatric liver transplantation: implications for quality of life and beyond. Pediatr Transplant. 2012;16:688-691.

12. Foulkes LM, Boggs SR, Fennell RS, Skibinski K. Social support, family variables, and compliance in renal transplant children. Pediatr Nephrol. 1993;7:185-188.

13. Gerson AC, Furth SL, Neu AM, Fivush BA. Assessing associations between medication adherence and potentially modifiable psychosocia variables in pediatric kidney transplant recipients and their families. Pediatr Transplant. 2004:8:543-550.

14. Guilfoyle SM, Goebel JW, Pai AL. Efficacy and flexibility impact perceived adherence barriers in pediatric kidney post-transplantation. Fam Syst Health. 2011;29:44-54.

15. Simons LE, Blount RL. Identifying barriers to medication adherence in adolescent transplant recipients. J Pediatr Psychol. 2007;32:831-844.

16. Soliday E, Kool E, Lande MB. Family environment, child behavior, and medical indicators in children with kidney disease. Child Psychiat Hum D. 2001;31:279-295.

17. Pai AL, Greenley RN, Lewandowski A, Drotar D, Youngstrom $\mathrm{E}$, Peterson CC. A meta-analytic review of the influence of pediatric cancer on parent and family functioning. J Fam Psychol. 2007;21:407-415.

18. Rubin RR, Peyrot M. Psychosocial problems and interventions in diabetes: a review of the literature. Diabetes Care. 1992;15:1640-1657.

19. Lewandowski AS, Palermo TM, Stinson J, Handley S, Chambers CT. Systematic review of family functioning in families of children and adolescents with chronic pain. J Pain. 2010;11:1027-1038.

20. Higgins JPT, Green S. Cochrane Handbook for Systematic Reviews of Interventions. New York: Wiley Online Library; 2011.

21. Zelikovsky N, Schast AP, Jean-Francois D. Parent stress and coping: waiting for a child to receive a kidney transplant. J Clin Psychol Med S. 2007;14:320-329.

22. Douglas JE, Hulson B, Trompeter RS. Psycho-social outcome of parents and young children after renal transplantation. Child Care Hlth Dev. 1998;24:73-84.

23. Young GS, Mintzer LL, Seacord D, Castañeda M, Mesrkhani V, Stuber ML. Symptoms of posttraumatic stress disorder in parents of transplant recipients: incidence, severity, and related factors. Pediatrics. 2003;111:725-731

24. Tarbell SE, Kosmach B. Parental psychosocial outcomes in pediatric liver and/or intestinal transplantation: pretransplantation and the early postoperative period. Liver Transplant Sur. 1998;4:378-387.

25. Diseth TH, Tangeraas T, Reinfjell T, Bjerre A. Kidney transplantation in childhood: mental health and quality of life of children and caregivers. Pediatr Nephrol. 2011;26:1881-1892.

26. Simons L, Ingerski LM, Janicke DM. Social support, coping, and psychological distress in mothers and fathers of pediatric transplant candidates: a pilot study. Pediatr Transplant. 2007;11:781-787.
27. Ingerski LM, Shaw K, Gray WN, Janicke DM. A pilot study comparing traumatic stress symptoms by child and parent report across pediatric chronic illness groups. J Dev Behav Pediatr. 2010;31:713-719.

28. Farley LM, DeMaso DR, D'Angelo E, et al. Parenting stress and parental post-traumatic stress disorder in families after pediatric heart transplantation. J Heart Lung Transpl. 2007;26:120-126.

29. Anthony SJ, Hebert D, Todd L, et al. Child and parental perspectives of multidimensional quality of life outcomes after kidney transplantation. Pediatr Transplant. 2010;14:249-256.

30. Rodrigue JR, MacNaughton K, Hoffmann RG III, et al. Transplantation in children: a longitudinal assessment of mothers' stress, coping, and perceptions of family functioning. Psychosomatics. 1997;38:478-486.

31. Uzark KC, Crowley DC. Family stress after pediatric heart transplantation. Prog Cardiovasc Nurs. 1989;4:23-27.

32. Uzark KC, Sauer SN, Lawrence KS, Miller J, Addonizio L, Crowley DC. The psychosocial impact of pediatric heart transplantation. J Heart Lung Transpl. 1992;11:1160-1167.

33. Fedewa MM, Oberst MT. Family caregiving in a pediatric renal transplant population. Pediatr Nursing. 1996;22:402-409.

34. LoBiondo-Wood G, Williams L, Kouzekanani K, McGhee C. Family adaptation to a child's transplant: pretransplant phase. Prog Transplant. 2000;10:81-87.

35. Suddaby E, Flattery M, Luna M. Stress and coping among parents of children awaiting cardiac transplantation. J Transpl Coord. 1997;7:36-40.

36. Rodrigue JR, MacNaughton K, Hoffmann RG III, et al. Perceptions of parenting stress and family relations by fathers of children evaluated for organ transplantation. Psychol Rep. 1996;79:723-727.

37. Lerret SM, Weiss ME. How ready are they? Parents of pediatric solid organ transplant recipients and the transition from hospital to home following transplant. Pediatr Transplant. 2011;15:606-616.

38. DeBolt AJ, Stewart SM, Kennard BD, Petrik K, Andrews WS. A survey of psychosocial adaptation in long-term survivors of pediatric liver transplants. Child Health Care. 1995;24:79-96.

39. Splinter K, Niemi AK, Cox R, et al. Impaired health-related quality of life in children and families affected by methylmalonic acidemia. $J$ Gene Couns. 2015;5:1-9.

40. Kaller T, Petersen I, Petermann F, Fischer L, Grabhorn E, Schulz KH. Family strain and its relation to psychosocial dysfunction in children and adolescents after liver transplantation. Pediatr Transplant. 2014;18:851-859.

41. Fukunishi I, Kudo H. Psychiatric problems of pediatric end-stage renal failure. Gen Hosp Psychiat. 1995;17:32-36.

42. Soliday E, Kool E, Lande MB. Psychosocial adjustment in children with kidney disease. J Pediatr Psychol. 2000;25:93-103.

43. Alonso EM, Neighbors K, Barton FB, et al. Health-related quality of life and family function following pediatric liver transplantation. Liver Transplant. 2008;14:460-468.

44. Sanchez C, Eymann A, De Cunto C, D'Agostino D. Quality of life in pediatric liver transplantation in a single-center in South America. Pediatr Transplant. 2010;14:332-336.

45. Stone RD, Beasley PJ, Treacy SJ, Twente AW, Vacanti JP. Children and families can achieve normal psychological adjustment and a good quality of life following pediatric liver transplantation: a long-term study. Transplant Proc. 1997;29:1571-1572.

46. Kikuchi R, Ono M, Kinugawa $\mathrm{K}$, et al. Health-related quality of life in parents of pediatric solid organ transplant recipients in Japan. Pediatr Transplant. 2015;19:332-341.

47. DeMaso DR, Kelley SD, Bastardi H, O'Brien P, Blume ED. The longitudinal impact of psychological functioning, medical severity, and family functioning in pediatric heart transplantation. J Heart Lung Transpl. 2004;23:473-480.

48. Devine KA, Reed-Knight B, Loiselle KA, Simons LE, Mee LL, Blount RL. Predictors of long-term health-related quality of life in adolescent solid organ transplant recipients. J Pediatr Psychol. 2011;36: 891-901. 
49. Denny B, Beyerle K, Kienhuis M, Cora A, Gavidia-Payne S, Hardikar W. New insights into family functioning and quality of life after pediatric liver transplantation. Pediatr Transplant. 2012;16:711-715.

50. Mavis AM, Ertl A, Chapman S, Cassidy LD, Lerret SM. Vulnerability and chronic illness management in pediatric kidney and liver transplant recipients. Prog Transplant. 2015;25:139-146.

51. Lerret SM, Weiss ME, Stendahl GL, et al. Pediatric solid organ transplant recipients: transition to home and chronic illness care. Pediatr Transplant. 2015;19:118-129.

52. Posfay-Barbe KM, Barbe RP, Wetterwald R, Belli DC, McLin VA. Parental functioning improves the developmental quotient of pediatric liver transplant recipients. Pediatr Transplant. 2013;17:355-361.

53. Dew MA, Myaskovsky L, Dimartini AF, Switzer GE, Schulberg $\mathrm{HC}$, Kormos RL. Onset, timing and risk for depression and anxiety in family caregivers to heart transplant recipients. Psychol Med. 2004;34:1065-1082.

54. Rodrigue JR, Dimitri N, Reed A, Antonellis T, Hanto DW, Curry M. Quality of life and psychosocial functioning of spouse/partner caregivers before and after liver transplantation. Clin Transplant. 2011;25:239-247.

55. Kessler RC, Chiu WT, Demler O, Merikangas KR, Walters EE. Prevalence, severity, and comorbidity of 12-month DSM-IV disorders in the National Comorbidity Survey Replication. Arch Gen Psychiatry. 2005;62:617-627.

56. Kazak AE, Alderfer M, Rourke MT, Simms S, Streisand R, Grossman JR. Posttraumatic stress disorder (PTSD) and posttraumatic stress symptoms (PTSS) in families of adolescent childhood cancer survivors. $J$ Pediatr Psychol. 2004;29:211-219.

57. Celano M, Klinnert MD, Holsey CN, McQuaid EL. Validity of the family asthma management system scale with an Urban African-American sample. J Pediatr Psych. 2011;36:576-585.

58. Eddy ME, Carter BD, Kronenberger WG, et al. Parent relationships and compliance in cystic fibrosis. J Pediatr Health Car. 1998;12:196-202.

59. Logan DE, Radcliffe J, Smith-Whitley K. Parent factors and adolescent sickle cell disease: associations with patterns of health service use. J Pediatr Psych. 2002;27:475-484.

60. Racine NM, Riddell RR, Khan M, Calic M, Taddio A, Tablon P. Systematic review: predisposing, precipitating, perpetuating, and present factors predicting anticipatory distress to painful medical procedures in children. J Pediatr Psych. 2016;41:159-181.

61. Dahlquist LM, Shroff Pendley J, Power TG, Landthrip DS, Jones CL, Steuber CP. Adult command structure and children's distress during the anticipatory phase of invasive cancer procedures. Child Health Care. 2001;30:151-167.

62. Streisand R, Swift E, Wickmark T, Chen R, Holmes CS. Pediatric parenting stress among parents of children with type 1 diabetes: the role of self-efficacy, responsibility, and fear. J Pediatr Psych. 2005;30:513-521.

63. Vrijmoet-Wiersma CM, Hoekstra-Weebers JE, Margreet de Peinder WM, et al.Psychometric qualities of the Dutch version of the pediatric inventory for parents (PIP): a multi-center study. Psycho-Oncology. 2010;19:368-375.

64. Hentinen M, Kyngäs $\mathrm{H}$. Factors associated with the adaptation of parents with a chronically ill child. J Clin Nurs. 1998;7:316-324.

65. Pai AL, Tackett A, Ittenbach RF, Goebel J. Psychosocial Assessment Tool 2.0_General: validity of a psychosocial risk screener in a pediatric kidney transplant sample. Pediatr Transplant. 2012;16:92-98.

66. Fredericks E, Smith T, Sevecke J, et al. Use of the Psychosocial Assessment Tool to identify risk in families of pediatric liver transplant recipients. Am J Transplant. 2016;16(Suppl. 3).

67. Kazak AE, Simms S, Barakat L, et al. Surviving Cancer Competently Intervention Program (SCCIP): a cognitive-behavioral and family therapy intervention for adolescent survivors of childhood cancer and their families. Fam Process. 1999;38:176-191.

68. Chou CY, Chen YC, Chen CL, Chen JL, Mu PF. Family experience of waiting for living donor liver transplantation: from parental donor perspective. J Clin Nurs. 2009;18:1684-1692.

69. Mendes AM, Bousso RS. Not being able to live like before: the family dynamics during the experience of pediatric liver transplantation. Revista Latino-Americana de Enfermagem. 2009;17:74-80.

70. Williams L, Eilers J, Heermann J, Smith K. The lived experience of parents and guardians providing care for child transplant recipients. Prog Transplant. 2012;22:393-402.

How to cite this article: Cousino MK, Rea KE, Schumacher KR, Magee JC, Fredericks EM. A systematic review of parent and family functioning in pediatric solid organ transplant populations. Pediatr Transplant. 2017;21:e12900.

https://doi.org/10.1111/petr.12900 